\title{
Nuclear theory
}

A Unified Theory of the Nucleus. By K. Wildermuth and Y. C. Tang. Pp. $x+389$. (Academic: London and New York, 1977.) $\$ 38.50 ; £ 27.35$.

$I_{N}$ nuclear theory the essential problem is to reduce the description of a manyparticle system to manageable proportions without the introduction of too many arbitrary assumptions and with the possibility of estimating the effects of approximations made. In the unified theory of nuclear reactions, particularly associated with the name of Feshbach. projection operators are used to reduce the true many-particle Hamiltonian to effective Hamiltonians, which belong to subspaces of the total Hilbert space but contain the correct asymptotic boundary conditions for the final states. In contrast, Wildermuth and Tang propose a theory in which the total wavefunction for the many-particle system is represented as a sum of nonorthogonal terms each corresponding to a final system with the correct boundary conditions. Thus, the subspaces of the total Hilbert space are defined through the wavefunction and. it is argued, this provides the correct asymptotic behaviour for the initial state and all final states.

The authors apply their approach to a variety of bound-state and reaction problems. The terms of the wavefunction each correspond to a possible cluster structure of the many-particle system, although, as the authors repeatedly stress, it is inappropriate to take these cluster structures too literally because of the effect of the antisymmetrisation operator which exchanges particles between the clusters. Among the problems discussed are the construction of bound-state wavefunctions and the evaluation of charge density distributions, ground-state energies and transition probabilities, the scattering of light systems at low and medium energies, resonance reactions, the microscopic formulation of optical potentials, and fission.

This book covers a very wide range of nuclear physics and contains some penetrating observations about aspects of the theory. It is, however, a rather frustrating book to read. So many topics are started and reach a premature conclusion with a rather trivial example based on a very light system such as ${ }^{8} \mathrm{Be}$ or ${ }^{6} \mathrm{Li}$. There is a useful discussion of the generator coordinate technique but recent developments in the resonating group method and the orthogonality condition model are ignored. The discussion of optical potentials for composite particles completely ignores extensive work done with folding models. The most serious weakness of the approach is that, although great emphasis is laid on the

\section{Cancer chemotherapy}

Biosynthetic Products for Cancer Chemotherapy. Vol. 1. By G. R. Pettit. Pp. xii +215 . (Plenum: New York and London, 1977.) \$23.40.

THE author states that his main aim is "to provide a current overall view of the cancer problem and the development of cancer chemotherapeutic drugs of biosynthetic origin". If you want a reasonably detailed summary of the chemical and biological properties of the kinds of cytotoxic products which have been isolated from plants, microorganisms, marine organisms, reptiles and other sources, then this book, from p47 on, may supply your needs.

It is less easy to know for whom the whole book was written. The first chapter (Introduction and Perspective) is the longest (46 pages); in it, the author discusses the nature, causation, and treatment of cancer with special emphasis on the role of the National Cancer Institute. Such a survey seems out of place and belies the title of the book. Dr Pettit could undoubtedly write a useful review or two on different aspects of cancer chemotherapy, but not, as here, by trying to cram a quart into an inappropriate pint pot.

In his introductory chapter, the author refers to the ten or so malignant conditions particularly amenable to drug treatment, without making it clear that these are relatively rare cancers. Their control is a commendable achievement, but is only a scratch on the surface of the cancer problem.

On the credit side, the author and publishers are to be complimented on the clear and numerous structural formulae. They occupy, however, all
D. F. Jackson

need for correct asymptotic behaviour of wavefunctions, all the examples fall back on oscillator functions which are notoriously incorrect at large separation distances. Until the practical application of the theory can be extended to yield the correct asymptotic behaviour the formal theory is unlikely to lead to major advance.

D. F. Jackson is Professor of Physics at the University of Surrey, UK. of 80 pages, leaving only about 75 pages of text for the natural products section of the book. Dr Pettit has succeeded in compressing a large amount of information into these pages, and the comprehensive tabular survey which volume 2 will provide, promises to be a useful supplement.

The author's. enthusiasm often comes through, insufficiently tempered, perhaps, by an acknowledgement of the limitations of cancer chemotherapy in man at the present time. He clearly feels that much more money could usefully be spent on the exploration of natural products as potential antitumour agents. He may be right, but some of his statements would not find universal acceptance. Take, for example, the dogmatic comment on p175: "Given sufficient financial support and time, the lower animals will prove to be a particularly good source of unusual and valuable drugs for cancer chemotherapy". Again, the author seems to imply (p58) that, with sufficient knowledge, one could design a single substance effective against human cancer in its various forms, a view now held by few.

Such criticism does not invalidate the work as a handy reference book on a range of naturally occurring cytotoxic compounds, although-if the reviewer may now be dogmatic-the very great majority of these products will never become established as clinically useful drugs. One must therefore not take the title of the book too literally.

J. A. Stock

J. A. Stock is Senior Lecturer in Chemistry at the Chester Beatty Institute of Cancer Research, London, UK. 